\title{
Measuring the fading of So galaxies using globular clusters
}

\author{
A. Aragón-Salamanca, A. G. Bedregal, and M. R. Merrifield \\ School of Physics and Astronomy, University of Nottingham, NG7 2RD, UK
}

Received 30 June 2006 / Accepted 25 July 2006

\begin{abstract}
Aims. We test the hypothesis that S0 galaxies are the descendants of fading spirals whose star formation has been shut down, by using the properties of their globular cluster systems.

Methods. We estimate the amount by which the globular cluster specific frequency (number of globular clusters per unit $V$-band luminosity) is enhanced in S0s relative to spirals. If the transformation hypothesis is correct, and no clusters are created or destroyed in the process, then this difference provides a measure of the degree to which the S0's $V$-band luminosity has faded relative to that of its spiral progenitor, which we can compare with the independent values estimated from stellar population synthesis and the $\mathrm{S} 0$ Tully-Fisher relation. We also explore whether the degree to which the globular cluster specific frequency is enhanced in S0s correlates with the colour of the stellar population, as also predicted by this hypothesis in which galaxies become redder as they fade.

Results. We find that, on average, the globular cluster specific frequency is a factor $\sim 3$ larger for S0s than for spirals, which can be interpreted as meaning that passively-evolving S0s have faded on average by about a factor of three from their spiral progenitors. This value fits remarkably well with the predictions of stellar population synthesis calculations, and the offset between the S0 and spiral Tully-Fisher relations, where the So $V$-band relation lies $\sim 1.2 \mathrm{mag}$, or a factor of three, below the spiral relation. We also find that the global colours of S0 galaxies are strongly correlated with their globular cluster specific frequencies: the redder the stellar population of an S0, the larger its specific frequency, as we might expect if we are catching different S0s at different stages of passively fading and reddening. Comparison to the predictions of stellar population synthesis models show that this explanation works quantitatively as well as qualitatively.

Conclusions. These tests strongly support the hypothesis that S0 galaxies were once normal spirals, whose star formation was cut off, presumably due to a change of environment. We are now in a position to start to make quantitative measurements of when this life-changing event occurred in different galaxies.
\end{abstract}

Key words. galaxies: formation - galaxies: evolution - galaxies: elliptical and lenticular, cD - galaxies: spiral - galaxies: star clusters

\section{Introduction}

S0, or lenticular, galaxies live at the crossroads between elliptical and spiral galaxies in the traditional Hubble tuning-fork diagram, suggesting that they should play a key role in understanding the morphologies of galaxies. In fact, in some cluster environments, S0s are the single most common type of luminous galaxy (Dressler 1980), so clearly understanding how they form and evolve is essential if we wish to have a complete picture of how galaxy morphology is related to galaxy formation and the environment. Dressler (1980) also showed that as the fraction of S0s increases in the densest cluster environments, so the fraction of spirals decreases, naturally suggesting that S0s are simply "dead" spiral galaxies that have had their star formation shut off by their surroundings, and are now quietly fading away as their stellar populations age. Indeed, a number of mechanisms have been suggested that might cause such a transformation: ram-pressure stripping of disk gas could remove the raw material of the next stellar generation (Gunn \& Gott 1972), while the somewhat gentler removal of a larger-scale reservoir of halo gas could lead to a slower "strangulation" of star formation (Larson et al. 1980).

Although this scenario is quite plausible, we need some more direct evidence that it has actually occurred. Circumstantial evidence for such a transformation over cosmic timescales comes from observations which indicate that the proportion of S0 galaxies is substantially smaller in distant clusters than in nearby ones, while spirals show the opposite trend (see, for example, Dressler et al. 1997), but any inferences drawn from such studies always face the criticism that one may not be equating comparable systems at different redshifts. Further evidence that SOs are fading spirals comes from studies of the Tully-Fisher relation between luminosity and rotation speed for S0 galaxies, which is found to be rather broad and offset from the relation for spirals in the sense that the S0s are systematically fainter than the spirals. A recent analysis by Bedregal et al. (2006) found that the S0 relation in the $B$-band is offset by $\sim 1.4$ mag from the Sakai et al. (2000) relation for spirals, with a scatter of $\sim 1 \mathrm{mag}$. Such an offset can most straightforwardly be interpreted as arising from the fading of the S0s relative to their spiral progenitors, with the spread in the relation arising from the different epochs at which this fading commenced. Indeed, Bedregal et al. (2006) were able to uncover some evidence that the magnitude of each S0's offset from the spiral Tully-Fisher relation depends on the time since star formation was cut off, as measured by the age of its stellar population, just as this picture would predict. However, the evidence is still uncomfortably circumstantial, as we have had to assume that the ancient progenitors of these S0s respect the same Tully-Fisher relation that we see in the nearby spirals, and that the difference between the relations for S0s and spirals arises from luminosity evolution: the offset could also arise because these systems have fundamentally different mass properties, shifting the relation in rotation speed. 
Even with these assumptions, we can only tie spirals and S0s together on a statistical basis, as we do not have a direct connection between any $\mathrm{S} 0$ and the properties of its individual progenitor.

Ideally, we would like to find some historical record preserved in the current properties of an S0 that tells us about its luminosity during its early life as a spiral, and that will not have been defaced by the transformation process or any subsequent evolution of the galaxy. Fortunately, just such a record exists in the globular cluster (GC) population of the S0. As we will see below, GCs have a reasonably well defined specific frequency (number per unit galaxy $V$-band luminosity) for spiral galaxies, so the number of GCs provides a useful proxy for a spiral galaxy's luminosity. Further, the transformation from spiral to S0 is unlikely to alter the number of GCs significantly: the hydrodynamic processes that strip out the gas from the spiral will not have any impact on these collisionless stellar clusters, and the conversion is likely to be sufficiently benign that we are unlikely to lose clusters or produce any new ones as we find in more dramatic phenomena like mergers (Ashman \& Zepf 1998). In addition, the GCs are sufficiently old that their own passive fading will be very slow, so the observed number counts should not decrease significantly during the transformation. Thus, the number of GCs in an S0 offers a reasonably robust indicator of its spiral progenitor's luminosity, so the specific frequency of GCs in such a system provides a direct measure of the ratio of its progenitor's luminosity to its current luminosity, which we can compare to the less direct indicators of fading such as those provided by the Tully-Fisher relation.

In this paper, we carry out such an analysis, comparing the specific frequencies of GCs in S0s to those in spirals in Sect. 2 to see how much these systems must have faded, and going on to look for evidence that different amounts of fading might be related to different epochs of transformation in Sect. 3 . Conclusions are presented in Sect. 4.

\section{The specific frequency of globular clusters in SOs and spirals}

The specific frequency of GCs in a galaxy, $S_{N}$, is usually defined relative to the galaxy's $V$-band luminosity, normalized to $M_{V}=-15$ :

$S_{N}=N_{\mathrm{t}} 10^{-0.4\left(M_{V}^{\mathrm{T}}+15\right)}$

where $N_{\mathrm{t}}$ is the total number of GCs and $M_{V}^{\mathrm{T}}$ is the total absolute magnitude of the underlying galaxy (Harris \& van den Bergh 1981). Since $S_{N} \propto N_{\mathrm{t}} / L_{V}$, as discussed above, we would expect $S_{N}$ to grow as spiral galaxies fade into S0s, if, indeed, they do. Thus, by comparing the specific frequency of GCs in spirals and S0s, it should be possible to check whether $S_{N}$ is larger in S0s than in spirals as this scenario requires, and to estimate by how much on average spirals fade during this transformation, for comparison with less direct indicators like the Tully-Fisher relation.

Unfortunately, data sets of GC specific frequencies in galaxies with different morphologies do not exist for any reasonablycomplete sample of galaxies. However, two entirely independent compilations have been constructed, so we can make some assessment of the likely systematic uncertainties that may arise from any selection biases by comparing the results from these two data sets.

First, we have analyzed the values published by Ashman \& Zepf (1998), excluding dwarf galaxies $\left(M_{V}>-17\right)$ and cDs. The relevant data for spirals and S0s are summarized in Table 1.
Table 1. Spiral and S0 absolute magnitudes and global globular cluster specific frequencies from Ashman \& Zepf (1998).

\begin{tabular}{lcclcc}
\hline \hline \multicolumn{3}{c}{ Spiral Galaxies } & \multicolumn{3}{c}{ S0 Galaxies } \\
Name & $M_{V}^{T}$ & $S_{N}$ & Name & $M_{V}^{T}$ & $S_{N}$ \\
\hline MW & -21.3 & $0.5 \pm 0.1$ & NGC 524 & -22.5 & $3.3 \pm 1.0$ \\
LMC & -18.4 & $0.6 \pm 0.2$ & NGC 1387 & -20.4 & $2.7 \pm 0.8$ \\
M31 & -21.8 & $0.9 \pm 0.2$ & NGC 1553 & -21.3 & $1.4 \pm 0.3$ \\
M33 & -19.4 & $0.5 \pm 0.2$ & NGC 3115 & -21.3 & $1.6 \pm 0.4$ \\
NGC 253 & -20.2 & $0.2 \pm 0.1$ & NGC 3384 & -20.4 & $0.9 \pm 0.5$ \\
NGC 3031 & -21.1 & $0.7 \pm 0.1$ & NGC 3607 & -20.9 & $3.5 \pm 2.6$ \\
NGC 2683 & -20.8 & $1.7 \pm 0.5$ & NGC 4340 & -19.9 & $8.5 \pm 3.1$ \\
NGC 4565 & -21.5 & $0.5 \pm 0.1$ & NGC 4526 & -21.4 & $7.4 \pm 2.2$ \\
NGC 4216 & -21.8 & $1.2 \pm 0.6$ & & & \\
NGC 4569 & -21.7 & $1.9 \pm 0.6$ & & & \\
NGC 4594 & -22.2 & $2.0 \pm 1.0$ & & & \\
NGC 5170 & -21.6 & $0.9 \pm 0.3$ & & & \\
NGC 7814 & -20.4 & $3.5 \pm 1.1$ & & & $3.7 \pm 1.1$ \\
\hline Average & \multicolumn{3}{c}{$1.2 \pm 0.3$} & &
\end{tabular}
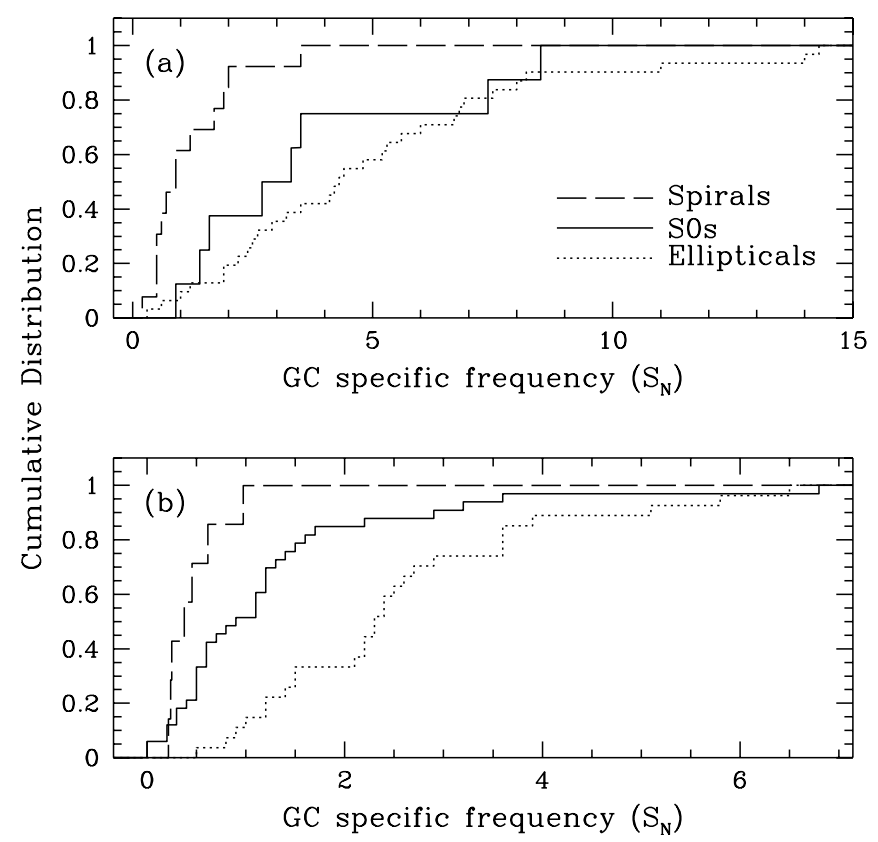

Fig. 1. a) Cumulative distribution of global GC specific frequencies for spiral, S0 and elliptical galaxies from Ashman \& Zepf (1998). b) Cumulative distribution of local GC specific frequencies for spiral, S0 and elliptical galaxies from Kundu \& Whitmore (2001a,b) and Goudfrooij et al. (2003).

In Fig. 1a, we plot the cumulative distributions of GC specific frequencies determined for spirals and S0s. For completeness, we also show the distribution for elliptical galaxies, but these have no direct bearing on the current analysis. It is immediately apparent that S0s have, on average, significantly larger specific frequencies than spirals. A Kolmogorov-Smirnov test rejects the hypothesis that spirals and S0s have the same $S_{N}$ distribution at the $90 \%$ confidence level, and we find that for this sample

$\frac{\left\langle S_{N}^{\mathrm{S} 0}\right\rangle}{\left\langle S_{N}^{\mathrm{Sp}}\right\rangle}=3.1 \pm 1.1$

where $\left\langle S_{N}^{\mathrm{Sp}}\right\rangle$ and $\left\langle S_{N}^{\mathrm{S} 0}\right\rangle$ are the unweighted average GC specific frequencies for spirals and SOs respectively. The uncertainties on the specific frequencies were calculated from the standard error in the mean, and these uncertainties were propagated to provide the quoted error in Eq. (2). 
Table 2. Absolute magnitudes and globular cluster specific frequencies from Kundu \& Whitmore (2001b) for S0s, and Goudfrooij et al. (2003) for spiral galaxies, with global colours for S0s where available.

\begin{tabular}{|c|c|c|c|c|c|c|c|}
\hline Name & $M_{V}^{T}$ & $S_{N}^{\text {global }}$ & $S_{N}^{\text {local }}$ & $(U-B)$ & $(B-V)$ & $(V-R)$ & $(V-I)$ \\
\hline \multicolumn{8}{|c|}{ Spiral Galaxies } \\
\hline NGC 3628 & -21.0 & $1.9 \pm 0.2$ & $1.0 \pm 0.1$ & - & - & - & - \\
\hline NGC 4013 & -20.8 & $1.1 \pm 0.3$ & $0.6 \pm 0.2$ & - & - & - & - \\
\hline NGC 4517 & -21.6 & $0.6 \pm 0.2$ & $0.2 \pm 0.1$ & - & - & - & - \\
\hline NGC 4565 & -21.5 & $0.6 \pm 0.2$ & $0.2 \pm 0.1$ & - & - & - & - \\
\hline NGC 4594 & -22.2 & $1.7 \pm 0.6$ & $0.4 \pm 0.2$ & - & - & - & - \\
\hline IC 5176 & -21.1 & $0.5 \pm 0.1$ & $0.2 \pm 0.1$ & - & - & - & - \\
\hline NGC 7814 & -20.5 & $0.7 \pm 0.2$ & $0.5 \pm 0.2$ & - & - & - & - \\
\hline Average & & & $0.4 \pm 0.1$ & & & & \\
\hline \multicolumn{8}{|c|}{ S0 Galaxies } \\
\hline NGC 524 & -22.4 & - & $1.1 \pm 0.4$ & - & - & - & - \\
\hline NGC 2768 & -22.0 & - & $1.2 \pm 0.4$ & - & - & - & - \\
\hline NGC 6861 & -21.8 & - & $3.6 \pm 1.6$ & $0.64 \pm 0.03$ & $1.04 \pm 0.03$ & $0.61 \pm 0.03$ & $1.31 \pm 0.03$ \\
\hline NGC 6703 & -21.8 & - & $1.2 \pm 0.8$ & - & - & - & - \\
\hline NGC 1553 & -21.5 & - & $0.5 \pm 0.1$ & $0.49 \pm 0.03$ & $0.95 \pm 0.03$ & $0.57 \pm 0.03$ & $1.19 \pm 0.03$ \\
\hline NGC 474 & -21.4 & - & $0.7 \pm 0.5$ & - & - & - & - \\
\hline NGC 3115 & -21.3 & - & $1.3 \pm 0.1$ & $0.59 \pm 0.03$ & $0.99 \pm 0.03$ & $0.61 \pm 0.03$ & $1.27 \pm 0.03$ \\
\hline NGC 1332 & -21.2 & - & $2.2 \pm 0.7$ & $0.60 \pm 0.03$ & $1.03 \pm 0.03$ & $0.60 \pm 0.03$ & $1.30 \pm 0.03$ \\
\hline NGC 3414 & -21.0 & - & $1.6 \pm 0.6$ & $0.55 \pm 0.01$ & $0.87 \pm 0.01$ & $0.70 \pm 0.01$ & - \\
\hline NGC 4459 & -20.9 & - & $0.9 \pm 0.3$ & $0.47 \pm 0.01$ & $0.96 \pm 0.01$ & $0.55 \pm 0.01$ & $1.22 \pm 0.02$ \\
\hline NGC 1201 & -20.8 & - & $1.1 \pm 0.5$ & $0.54 \pm 0.03$ & $0.97 \pm 0.03$ & $0.57 \pm 0.03$ & $1.22 \pm 0.03$ \\
\hline NGC 1400 & -20.6 & - & $2.9 \pm 1.1$ & $0.61 \pm 0.03$ & $1.03 \pm 0.03$ & $0.65 \pm 0.03$ & $1.37 \pm 0.03$ \\
\hline NGC 3607 & -20.4 & - & $1.3 \pm 0.4$ & - & - & - & - \\
\hline NGC 4203 & -20.2 & - & $1.5 \pm 0.5$ & - & - & - & - \\
\hline NGC 2902 & -20.2 & - & $0.3 \pm 0.4$ & - & - & - & - \\
\hline NGC 3489 & -19.6 & - & $1.4 \pm 0.7$ & - & - & - & - \\
\hline NGC 4379 & -19.6 & - & $0.6 \pm 0.4$ & $0.39 \pm 0.01$ & $0.85 \pm 0.01$ & $0.61 \pm 0.01$ & $1.24 \pm 0.02$ \\
\hline NGC 1389 & -19.5 & - & $0.5 \pm 0.4$ & $0.43 \pm 0.01$ & $0.92 \pm 0.01$ & $0.56 \pm 0.01$ & $1.16 \pm 0.02$ \\
\hline NGC 3056 & -18.9 & - & $0.6 \pm 0.7$ & - & - & - & - \\
\hline NGC 3156 & -18.9 & - & $0.4 \pm 0.4$ & - & - & - & - \\
\hline IC 3131 & -18.9 & - & $0.2 \pm 0.4$ & - & - & - & - \\
\hline NGC 1375 & -18.8 & - & $0.5 \pm 0.7$ & $0.31 \pm 0.03$ & $0.81 \pm 0.03$ & $0.51 \pm 0.03$ & $1.06 \pm 0.03$ \\
\hline VCC 165 & -18.5 & - & $0.0 \pm 2.1$ & - & - & - & - \\
\hline NGC 3599 & -18.4 & - & $1.2 \pm 0.9$ & - & - & - & - \\
\hline NGC 2328 & -18.4 & - & $0.0 \pm 1.4$ & - & - & - & - \\
\hline NGC 4431 & -18.4 & - & $0.5 \pm 0.7$ & - & - & - & - \\
\hline IC 1919 & -18.2 & - & $1.1 \pm 1.7$ & - & - & - & - \\
\hline NGC 1581 & -18.2 & - & $0.2 \pm 0.9$ & $0.20 \pm 0.03$ & $0.73 \pm 0.03$ & $0.46 \pm 0.03$ & $1.06 \pm 0.03$ \\
\hline ESO 358-G059 & -18.0 & - & $0.6 \pm 1.3$ & - & - & - & - \\
\hline NGC 3870 & -17.9 & - & $0.8 \pm 1.3$ & - & - & - & - \\
\hline NGC 3115-DW1 & -17.9 & - & $6.8 \pm 2.4$ & - & - & - & - \\
\hline ESO 118-G034 & -17.6 & - & $0.3 \pm 2.0$ & - & - & - & - \\
\hline IC 3540 & -17.3 & - & $3.2 \pm 4.2$ & - & - & - & - \\
\hline NGC 4150 & -16.6 & - & $1.7 \pm 1.8$ & - & - & - & - \\
\hline Average & & & $1.2 \pm 0.2$ & & & & \\
\hline
\end{tabular}

We have constructed the second $S_{N}$ data set using the results published by Kundu \& Whitmore (2001a) for elliptical galaxies, Kundu \& Whitmore (2001b) for S0s, and Goudfrooij et al. (2003) for spiral galaxies. Again, dwarf galaxies have been excluded. The adopted data for spirals and S0s are presented in Table 2. One complication here is that Kundu and Whitmore published mainly "local" $S_{N}$ values, corresponding to one WFPC2 field, while Goudfrooij et al. give global values comparable to those described above. However, Kundu \& Whitmore (2001a,b, hereafter KW) give both local and global specific frequencies for 14 galaxies, so it is possible to calibrate the difference between these quantities, and how they might depend on the galaxies' absolute magnitude. A least-squares fit to these data yields a calibration of

$\frac{S_{N}^{\text {local }}}{S_{N}^{\text {global }}}=5.71+0.25 M_{V}^{\mathrm{T}}$ with a scatter of only 0.18 . Since we are primarily interested in population average values of $S_{N}$, and we are looking at variations between populations that amount to factors of several, this $\sim 20 \%$ uncertainty is completely negligible. Ideally, we would use this transformation to convert the local values from $\mathrm{KW}$ into global values. However, the calibration of this relation depends almost entirely on galaxies with absolute magnitudes $M_{V}<-20$, and a number of galaxies in the KW sample are fainter than this limit, so such a transformation would have significant uncertainties. Fortunately, all the spiral galaxies in the Goudfrooij et al. (2003) sample lie at brighter magnitudes, so we can use the relation to transform their quoted global values into local values, as shown in Table 2; since we are only interested in the ratio of specific frequencies, it does not matter whether the local or global values are employed as long as we use the same in both samples. In Fig. 1b we plot the cumulative distributions of GC local specific frequencies for spirals, SOs and ellipticals 
from this second compilation. Again, S0s have, on average, significantly larger specific frequencies than spirals (once again, a Kolmogorov-Smirnov test rejects the hypothesis that spirals and S0s have the same $S_{N}$ distribution at the $90 \%$ confidence level, giving a rejection from the combined data sets that is significant at the $99 \%$ level). For this second dataset, we find that the ratio of local specific frequencies is

$$
\frac{\left\langle S_{N}^{\mathrm{S} 0}\right\rangle}{\left\langle S_{N}^{\mathrm{Sp}}\right\rangle} \simeq 2.7 \pm 0.9
$$

Given that Eqs. (2) and (4) were derived from completely different data sets derived using different instruments using different analysis techniques applied by different authors, the agreement between the two values suggests that this result is reasonably robust, notwithstanding the incompleteness of the samples. It would appear that spirals have a specific frequency of GCs that is a factor of $\sim 3$ lower than that in S0s, with the fairly simple interpretation that the latter have held onto their GCs but faded by a factor of $\sim 3$ in transforming from the former.

Is this factor plausible? Well, population synthesis models based on Bruzual \& Charlot (2003) indicate that a galaxy with an initially steady star formation rate will fade by a factor of $\sim 3$ in the $V$ band in $\sim 3$ Gyr after complete truncation of its star formation. If the star formation decays more slowly (as through the strangulation mechanism), such fading could take 4-6 Gyr (see Shioya et al. (2004) for some model examples). These timescales are comparable to the look-back time to $z \sim 0.5$, which is where Dressler et al. (1997) found signs of the transition from spiraldominated to S0-dominated clusters. However, we would not expect all galaxies to undergo the transition at the same time, and presumably some fraction of the significant scatter in $S_{N}$ for S0s can be attributed to the different amount of fading that has taken place for different systems that underwent this transformation at different times, a point to which we return in Sect. 3. Nonetheless, it is heartening that the difference in the mean value of $S_{N}$ seems entirely consistent with the characteristic look-back time at which the transformation is believed to have predominantly occurred.

The difference also matches up to the results from the analysis of the Tully-Fisher relation. As mentioned above, Bedregal et al. (2006) found a $\sim 1.4 \mathrm{mag}$ offset between the $B$-band Tully-Fisher relation for S0s and that for spirals. Using the same passively-evolving stellar population models as described above, we find that such a fading in the $B$-band corresponds to a fading in the $V$-band of $\sim 1.2 \mathrm{mag}$, or a factor of $\sim 3$ decrease in $V$-band luminosity, just as found in the analysis of globular clusters. Although most reassuring, the exact agreement must be to an extent coincidental: Bedregal et al. (2006) found significant uncertainty in the offset in the Tully-Fisher relations depending on the exact calibration adopted, so the factor of $\sim 3$ predicted by the Tully-Fisher analysis could easily be a factor of two or four. The lack of a complete sample of S0s upon which to carry out this test also means that some residual uncertainty must remain in the absolute value of the mean fading of the population, due to unknown systematic effects in the way that the sample was selected.

Thus, although the mean degree of fading as derived from the globular cluster specific frequency is entirely consistent with the values predicted by population synthesis and those derived independently from the Tully-Fisher relation, the case would be even stronger if there were a more subtle test that goes beyond simple absolute shifts in the mean of the population.

\section{Correlation between globular cluster specify frequency and galaxy colours}

Happily, there is a differential test that we can apply to the GC specific frequency data that overcomes these uncertainties in both absolute magnitude and sample selection. As an individual system fades from spiral to S0, the specific frequency of GCs will go up as we have discussed above, and the colours of the galaxy should redden as the stellar population ages. Thus, we should expect to see a correlation between the measured values of $S_{N}$ and the S0s' colours, with different values corresponding to the degree to which the S0 has evolved away from its spiral progenitor. Further, we can compare the form of this relation to that predicted by the population synthesis calculations to see whether the correlation matches the model predictions in detail.

Unfortunately, the necessary data do not exist for all of the S0 galaxies for which $S_{N}$ values are available. The evolution in colour in such a fading population is relatively modest, so accurate photometry is required, and we are interested in the galaxies' global stellar populations, so need spatially-resolved photometry rather than just central values for colours. Such high-quality extended data are not readily available for many galaxies, but using the photometry of Poulain \& Nieto (1994) and the highest-quality data from the compilation of Prugniel \& Heraudeau (1998), we have been able to bring together reliable multi-colour photometry for 12 of the non-dwarf S0 galaxies from Kundu \& Whitmore (2001b), which we summarize in Table 2. These 12 galaxies appear to be a fair sub-sample of the original sample, in that there is no obvious bias in magnitude or the values of $S_{N}$. To obtain a measure of the global populations in these galaxies without unduly compromising the photometric accuracy, we have calculated the integrated colours for these systems within an aperture that corresponds to the $B$-band halflight radius of each galaxy, as tabulated in de Vaucouleurs et al. (1976).

Figure 2 shows the derived global $U-B, B-V, V-R$ and $V-I$ colours for these 12 S0 galaxies plotted against their GC specific frequencies. A clear correlation is observed in the sense predicted by the fading spiral hypothesis: the larger the GC specific frequency in an S0, the redder its stellar population.

Further, the relation is of exactly the right amplitude. Figure 2 shows the predictions of population synthesis codes for a fading model stellar population with solar metallicity and a standard initial mass function, which ceased forming stars more than $\sim 1$ Gyr ago - after this time, the colour evolution is not very sensitive to the detailed history of the star formation that took place before star formation ended, so the model predictions are fairly robust. To assess the uncertainty in the predictions, we computed models using the codes of both Bruzual \& Charlot (2003) and Worthey (1994), but, as is clear from Fig. 2, these uncertainties are also fairly negligible compared to the error bars on the data. In these calculations, the variation in colour with age comes directly from the models, and the changes in $S_{N}$ come from the corresponding variations in the model values of the $V$ band magnitude, using Eq. (1) and following the basic precept of this paper that the number of GCs does not change with time. The $x$-axis zero-point of the models is fixed so that for the typical $V-I$ colour of a star-forming spiral, $(V-I) \simeq 1.1$ (Fukugita et al. 1995), the models predict $S_{N} \simeq 0.4$, which is the average value of $S_{N}^{\text {local }}$ for the star-forming spirals in the Goudfrooij et al. (2003) sample (see Table 2). Once we have made this calibration to the local spirals, the zero-points of the $x$-axes in all the other plots, the amplitude of all the colours' variations with $S_{N}$, their slopes, and even their curvatures are entirely fixed by the stellar 

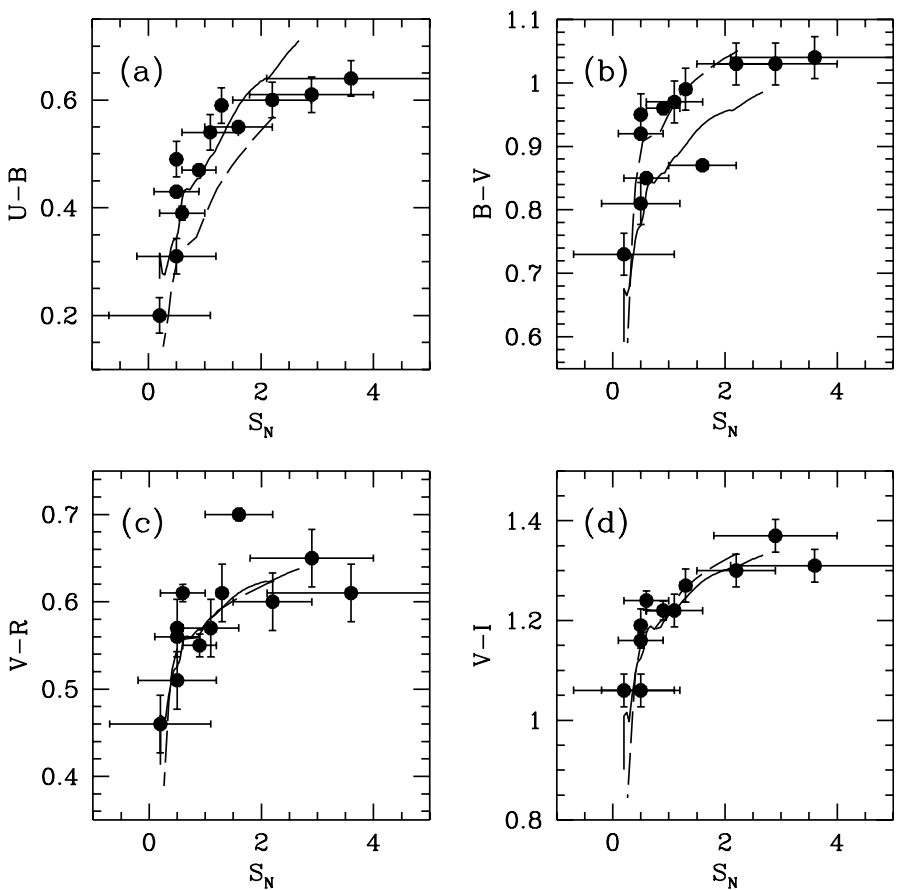

Fig. 2. Global $U-B, B-V, V-R$ and $V-I$ colours versus globular cluster specific frequency for S0 galaxies from Kundu \& Whitmore (2001b). The solid and and dashed lines correspond to population synthesis model predictions of fading stellar populations from Bruzual \& Charlot (2003) and Worthey (1994) respectively.

evolution models, and, as Fig. 2 attests, the match of all these properties to the observations is remarkably good.

\section{Conclusions}

The classic problem in trying to address questions related to galaxy evolution is that our snapshot view of the Universe means that we never get to view the process directly. Thus, although it seems quite plausible that spiral galaxies fade to become SOs when their interstellar media are stripped out and star formation ceases, there is little direct evidence to this effect. Here, we finesse this issue by making use of the historical record imprinted in a galaxy via its GC population, which should still be "readable" even after the transformation process. In particular, if the number of globular clusters is taken as a measure of the progenitor spiral galaxy's luminosity, and this number does not change as the galaxy passively evolves, then we have been able to show that S0s must have faded by an average factor of approximately three during this process. This result matches very neatly with the values predicted by stellar population synthesis models and those found by comparing the Tully-Fisher relations of spirals and S0s.

Further, using the amount by which the stellar population has reddened as a measure of the time since the transformation into an S0 began, we have been able to show that individual galaxies are at different stages along this evolutionary track: the colours of these galaxies correlate with the specific frequency of GCs in exactly the way that one would expect if their star formation shut down at different times in the past. Thus, we are at the point of being able to follow the histories of individual S0 galaxies with the detail necessary to say which underwent their life-changing transformations first.

The next logical step in writing the life histories of these galaxies is to obtain uniform samples of spectra of the quality necessary to derive stellar population ages for these systems (e.g. Kuntschner \& Davies 1998). Not only would these data resolve any remaining ambiguity by lifting the degeneracy between age and metallicity effects in the interpretation of the broad-band colours used here, but they would also allow us to put much more accurate dates to the "birthdays" of S0 galaxies.

Acknowledgements. We would like to thank Steven Bamford, Jean Brodie, Bo Milvang-Jensen, Osamu Nakamura and Frazer Pearce for very stimulating discussions. We would also like to thank the referee for suggestions that substantially improved the paper. MRM gratefully acknowledges the support of a PPARC Senior Fellowship.

\section{References}

Ashman, K. M., \& Zepf, S. E. 1998, Globular cluster systems (Cambridge: Cambridge Univ. Press)

Bedregal, A. G., Aragón-Salamanca, A., \& Merrifield, M. R. 2006, MNRAS, submitted

Bruzual, G., \& Charlot, S. 2003, MNRAS, 344, 1000

de Vaucouleurs, G., de Vaucouleurs, A., \& Corwin, H. G. 1976, Second reference catalogue of bright galaxies (University of Texas Press, Austin)

Dressler, A. 1980, ApJ, 236, 351

Dressler, A., Oemler, A. J., Couch, W. J., et al. 1997, ApJ, 490, 577

Fukugita, M., Shimasaku, K., \& Ichikawa, T. 1995, PASP, 107, 945

Goudfrooij, P., Strader, J., Brenneman, L., et al. 2003, MNRAS, 343, 665

Gunn, J. E., \& Gott, J. R. I. 1972, ApJ, 176, 1

Harris, W. E., \& van den Bergh, S. 1981, AJ, 86, 1627

Kundu, A., \& Whitmore, B. C. 2001a, AJ, 121, 2950

Kundu, A., \& Whitmore, B. C. 2001b, AJ, 122, 1251

Kuntschner, H., \& Davies, R. L. 1998, MNRAS, 295, L29

Larson, R. B., Tinsley, B. M., \& Caldwell, C. N. 1980, ApJ, 237, 692

Poulain, P., \& Nieto, J.-L. 1994, A\&AS, 103, 573

Prugniel, P., \& Heraudeau, P. 1998, A\&AS, 128, 299

Sakai, S., Mould, J. R., Hughes, S. M. G., et al. 2000, ApJ, 529, 698

Shioya, Y., Bekki, K., \& Couch, W. J. 2004, ApJ, 601, 654

Worthey, G. 1994, ApJS, 95, 107 